Louisiana State University

LSU Digital Commons

Faculty Publications

Department of Physics \& Astronomy

$12-20-2004$

\title{
CASTER - A scintillator-based black hole finder probe
}

\author{
M. L. McConnell \\ University of New Hampshire Durham \\ M. L. Cherry \\ Louisiana State University \\ T. G. Guzik \\ Louisiana State University \\ R. M. Kippen \\ Los Alamos National Laboratory \\ J. M. Ryan \\ University of New Hampshire Durham
}

See next page for additional authors

Follow this and additional works at: https://digitalcommons.Isu.edu/physics_astronomy_pubs

\section{Recommended Citation}

McConnell, M., Cherry, M., Guzik, T., Kippen, R., Ryan, J., Macri, J., Miller, R., Paciesas, W., Schaefer, B., Stacy, J., Wefel, J., \& Vestrand, W. (2004). CASTER - A scintillator-based black hole finder probe. Proceedings of SPIE - The International Society for Optical Engineering, 5488 (PART 2), 944-955. https://doi.org/10.1117/12.551602

This Conference Proceeding is brought to you for free and open access by the Department of Physics \& Astronomy at LSU Digital Commons. It has been accepted for inclusion in Faculty Publications by an authorized administrator of LSU Digital Commons. For more information, please contact ir@lsu.edu. 


\section{Authors}

M. L. McConnell, M. L. Cherry, T. G. Guzik, R. M. Kippen, J. M. Ryan, J. M. Macri, R. S. Miller, W. S. Paciesas,

B. E. Schaefer, J. G. Stacy, J. P. Wefel, and W. T. Vestrand 
University of New Hampshire

University of New Hampshire Scholars' Repository

Space Science Center

Institute for the Study of Earth, Oceans, and

Space (EOS)

10-11-2004

\title{
CASTER: a scintillator-based black hole finder probe
}

\author{
Mark L. McConnell \\ University of New Hampshire - Main Campus, mark.mcconnell@unh.edu \\ M L. Cherry \\ Louisiana State University - Baton Rouge \\ T G. Guzik \\ Louisiana State University - Baton Rouge \\ R M. Kippen \\ Los Alamos National Laboratory \\ James M. Ryan \\ University of New Hampshire, James.Ryan@unh.edu
}

See next page for additional authors

Follow this and additional works at: https://scholars.unh.edu/ssc

Part of the Astrophysics and Astronomy Commons

\footnotetext{
Recommended Citation

Mark L. McConnell ; Michael L. Cherry ; T. G. Guzik ; R. M. Kippen ; James M. Ryan ; John M. Macri ; Richard S. Miller ; William S. Paciesas ; Bradley E. Schaefer ; J. G. Stacy ; John P. Wefel and W. T. Vestrand "CASTER: a scintillator-based black hole finder probe", Proc. SPIE 5488, UV and Gamma-Ray Space Telescope Systems, 944 (October 11, 2004); doi:10.1117/12.551602; http://dx.doi.org/10.1117/ 12.551602

This Conference Proceeding is brought to you for free and open access by the Institute for the Study of Earth, Oceans, and Space (EOS) at University of New Hampshire Scholars' Repository. It has been accepted for inclusion in Space Science Center by an authorized administrator of University of New Hampshire Scholars' Repository. For more information, please contact Scholarly.Communication@unh.edu.
} 


\section{Authors}

Mark L. McConnell, M L. Cherry, T G. Guzik, R M. Kippen, James M. Ryan, John R. Macri, R S. Miller, W

Paciesas, B Schaefer, J G. Stacy, J P. Wefel, and W T. Vestrand 


\title{
CASTER - a scintillator-based Black Hole Finder Probe
}

\author{
M. L. McConnell ${ }^{\mathrm{a}}$, M. L. Cherry ${ }^{\mathrm{b}}$, T. G. Guzik ${ }^{\mathrm{b}}$, R. M. Kippen ${ }^{\mathrm{d}}$, J. M. Ryan ${ }^{\mathrm{a}}$, J. M. Macria \\ R. S. Miller, W. S. Paciesas, , B. E. Schaefer ${ }^{\mathrm{e}}$, J. G. Stacy, , J. P. Wefel ${ }^{\mathrm{b}}$, W. T. Vestrand \\ ${ }^{a}$ Space Science Center, University of New Hampshire, Durham, NH USA 03824 \\ ${ }^{\mathrm{b}}$ Department of Physics and Astronomy, Louisiana State University, Baton Rouge, LA USA 70803 \\ ${ }^{c}$ Department of Physics, Southern University, Baton Rouge, LA USA 70813 \\ ${ }^{\mathrm{d}}$ Los Alamos National Laboratory, Los Alamos, NM USA 87545 \\ ${ }^{\mathrm{e}}$ Department of Physics, University of Alabama, Huntsville, AL USA 35899
}

\begin{abstract}
The primary scientific mission of the Black Hole Finder Probe (BHFP), part of the NASA Beyond Einstein program, is to survey the local Universe for black holes over a wide range of mass and accretion rate. One approach to such a survey is a hard X-ray coded-aperture imaging mission operating in the $10-600 \mathrm{keV}$ energy band, a spectral range that is considered to be especially useful in the detection of black hole sources. The development of new inorganic scintillator materials provides improved performance (for example, with regards to energy resolution and timing) that is well suited to the BHFP science requirements. Detection planes formed with these materials coupled with a new generation of readout devices represent a major advancement in the performance capabilities of scintillator-based gamma cameras. Here, we discuss the Coded Aperture Survey Telescope for Energetic Radiation (CASTER), a concept that represents a BHFP based on the use of the latest scintillator technology.
\end{abstract}

Keywords: coded aperture imaging, gamma-rays, hard x-rays, lanthanum bromide, scintillator

\section{INTRODUCTION}

The primary scientific mission of the Black Hole Finder Probe (BHFP), part of the NASA "Beyond Einstein" program, is to survey the local Universe for black holes over a wide range of mass and accretion rate. One approach to such a survey is a hard X-ray coded-aperture imaging mission operating in the $10-600 \mathrm{keV}$ energy band, a spectral range that is considered to be especially useful in the detection of black hole sources. In support of such a mission, considerable attention has been devoted to the development of room-temperature solid-state spectrometers, particularly cadmium zinc telluride (CZT). CZT detectors promise excellent energy and spatial resolutions, and, in principle, satisfy the science requirements of a BHFP mission. However, given the cost and complexity issues associated with thick, large area CZT, it is prudent to consider alternative detector technologies. This is especially true, given that several new experimental techniques are successfully being applied using standard technologies, such as inorganic scintillators, wavelength-shifting fibers and photomultiplier tubes (PMTs), all of which have laboratory and space flight heritage. In addition, the development of a new inorganic scintillator material, lanthanum bromide $\left(\mathrm{LaBr}_{3}\right)$, provides improved performance that is well suited to the BHFP science requirements. Detection planes formed with $\mathrm{LaBr}_{3}$ scintillator coupled with a new generation of readout devices may represent a major advancement in the performance capabilities of scintillator-based gamma cameras. To help insure a timely BHFP mission, we are developing a mission concept based on the use of more traditional detector technologies.

The task of a hard X-ray BHFP mission is to perform an all-sky census of black hole sources with a 1 -year $5 \sigma$ sensitivity level of $\mathrm{F}_{\text {lim }} \sim 5 \times 10^{-13} \mathrm{erg} \mathrm{cm}^{-2} \mathrm{~s}^{-1}(20-100 \mathrm{keV})$. This corresponds to $\sim 0.02 \mathrm{mCrab}$ in the $20-100 \mathrm{keV}$ energy band, a sensitivity level that is comparable to that of the all-sky survey performed at lower energies $(0.5-2.5$ $\mathrm{keV}$ ) by ROSAT. As currently conceived, the BHFP will provide a hard X-ray survey that is $\sim 1000 \times$ more sensitive than the only previous all-sky survey (HEAO A-4), 1-20× more sensitive that the all-sky survey of Swift, and $\sim 20 \times$ more sensitive than CGRO/BATSE for $\gamma$-ray bursts. Given this sensitivity requirement, an angular resolution of 3-5 arcmin is necessary to avoid source confusion from the more than 30,000 AGN that will be detected. This will permit 
the localization of bright sources with an accuracy of $\sim 10$ arcsec. The faintest sources will have $\sim 1$ arcmin centroids, sufficient for identification with bright galaxies or as a guide for higher resolution instruments such as Con-X or HSI. These scientific objectives can only be met by using a coded-aperture imaging instrument. The scientific requirements lead to a set of detector constraints. Spatial resolution (in 3-dimensions), the ability to resolve multi-hit events, photon detection efficiency, photo-fraction and energy resolution are the most important detector performance characteristics. Cost and availability of detector material are important risk considerations.

The detector technology is an important driver for the mission design. For example, limitations on detector thickness (i.e., detector efficiency) dictate the requirement on detector area, with all its implications for experiment size, weight, power, etc. This in turn constrains the choices for launch vehicle and orbit. Scintillators are a proven technology that is robust, reliable and simple to implement in large areas and in large volumes. With $\mathrm{LaBr}_{3}$, we now have the prospect of scintillators with energy resolution and stopping power on par with CZT, but with far less cost. Although we have been and continue to be enthusiastically involved in the development of CZT detectors for astrophysics, we feel that one must pursue this alternative technology given the cost and complexity of solid-state detectors. We believe that all of the primary scientific objectives of the BHFP mission, as envisioned in the Beyond Einstein Roadmap, can be achieved with a coded aperture mission based on detector designs that employ inorganic scintillator. In addition, scintillator technology offers a practical means to extend the effective energy range beyond $511 \mathrm{keV}$, an important goal that will be difficult to achieve with CZT. We therefore propose a mission concept study to explore the implications, benefits and penalties, both practical and scientific, of using inorganic scintillators as the detector technology of a coded aperture imaging BHFP. For convenience, we will refer to our design concept as the Coded Aperture Survey Telescope for Energetic Radiation (CASTER).

\section{SCIENCE}

Although the 10-600 keV energy band encompasses a wide range of astrophysical phenomena, black hole sources are particularly prominent. Many accreting black hole sources (especially stellar-mass black holes and Seyfert galaxies) have spectra that peak in this energy band. This characteristic makes this particular energy range ideal for a BHFP mission. The all-sky nature of the mission, coupled with the large effective area, will provide an opportunity to monitor variable sources on a wide range of time scales, from milliseconds to years. The scientific goals of CASTER are closely aligned with those of the EXIST mission, a concept that is also being considered for the BHFP mission. Given that the scientific goals of the EXIST mission have been discussed extensively in the literature, ${ }^{1,2}$ we will not attempt here to provide an overview of all of the science potential for the BHFP mission. Instead, we will highlight some of the science that would be addressed at the higher end of the BHFP energy range ( $>200 \mathrm{keV})$, where the CASTER design may offer significant advantages.

Stellar mass black holes are perhaps the best understood. They are observable as a result of their accretion of matter from a normal-type stellar companion. The first black hole candidate ever discovered, Cygnus X-1, has been studied extensively throughout the entire electromagnetic spectrum. At high energies, its spectrum has been measured out to several MeV. ${ }^{3}$ Galactic black hole candidates (GBHC) are generally observed at these energies in one of two spectral states that can be characterized as either a "breaking $\gamma$-ray state" or a "power-law $\gamma$-ray state". 4 Studying the highenergy spectral variability of sources over an extended period of time will be one of the goals of CASTER. Being able to do so will require sensitivity out to $600 \mathrm{keV}$ in order to clearly distinguish between the two spectral states and to better define the precise spectral shapes. A search for emission at $511 \mathrm{keV}$ (perhaps redshifted), a potential tracer of pair plasmas, will also be possible.

The origin of the diffuse $511 \mathrm{keV}$ line emission seen by both CGRO/OSSE ${ }^{5}$ and INTEGRAL ${ }^{6}$ remains a mystery. Although there are several possible sources of the underlying positron population, there is, as yet, no clear consensus on the answer to this issue. ${ }^{7}$ It may be that the observed emission consists of numerous point sources (such as accreting stellar mass black holes or $\gamma$-ray bursts ${ }^{8,9}$ ) or it may be that the emission represents the annihilation of a new form of light dark matter (LDM). ${ }^{10-11}$ A clarification of this issue may have to await more sensitive observations with finer angular resolution. 
The CASTER mission will provide a $\gamma$-ray burst (GRB) monitoring capability that will be $\sim 20 \times$ more sensitive than CGRO/BATSE, which will lead to the most detailed population study of GRBs ever performed. It has been estimated ${ }^{12}$ that a coded-aperture BHFP mission will provide sub-arcmin locations for 2 - 3 GRBs per day, roughly twice the rate predicted for the upcoming Swift mission. This will permit rapid follow-up of GRBs by other contemporary missions such as Con-X and NGST, as well as numerous ground-based observatories. Correlated studies with LISA will shed light on the gravitational waves that are generated by a GRB. A carefully coordinated rapid response network will be required to maximize the return of GRB science. The $10-600 \mathrm{keV}$ energy band will be especially useful in that many GRB spectra peak in this energy range. Observations up to $\sim 600 \mathrm{keV}$ will be required in order to cover the full range of $E_{p}$ values. Performing spectral measurements that extend beyond $E_{p}$ will insure that the spectra are properly characterized.

The CASTER design (assuming the use of $\mathrm{LaBr}_{3}$ ) is comparable to Swift ${ }^{13}$ and INTEGRAL ${ }^{14}$ in terms of energy resolution. A very small fraction of sources detected by CASTER are expected to be candidates for line studies. Although CASTER will have very good line sensitivity and energy resolution, more detailed spectral studies (including line shapes) could be made in this energy range with one or more of several future instruments currently under development, such as HEFT,${ }^{15}$ InFOC $\mu \mathrm{s},{ }^{16} \mathrm{~B}-\mathrm{MINE},{ }^{17} \mathrm{CLAIRE},{ }^{18} \mathrm{HIS} / \mathrm{NuSTAR},{ }^{19}$ and CYCLONE. ${ }^{20}$

\section{IMPLEMENTATION APPROACH}

We are developing a BHFP mission concept using one or more wide-FoV coded aperture instruments with detection planes based on inorganic scintillator technology. One part of our study is to evaluate what can be accomplished using new experimental techniques with established scintillator material (e.g., segmented CsI). We are also considering the use of $\mathrm{LaBr}_{3}$, a relatively new scintillator material that offers significantly improved light output and energy resolution. Hybrid detector arrays (e.g., $\mathrm{LaBr}_{3}$ and CZT) may also be considered to optimize the instrument performance and sensitivity throughout the full $10-600 \mathrm{keV}$ energy range. Trade studies will be conducted using Monte Carlo simulations of candidate coded aperture instrument configurations (including mask, image plane, shield, etc.) to identify configurations meeting the science goals and to evaluate their viability given an assessment of technology readiness, the mission budget and the timeframe for the BHFP. Our goal is to find an achievable instrument configuration that meets the science goals and mission constraints using a low-background equatorial orbit.

\subsection{Coded aperture imaging}

Although other means of imaging high-energy radiation are possible, such as rotation modulation collimators or Fresnel zone plate imaging, only traditional coded aperture imaging ${ }^{21,22}$ offers the wide FoV that is needed for the full-sky survey of the BHFP. The coded mask must be thick enough to provide a high contrast shadow on the detector plane. The measured shadow is used to construct the sky image, so the ability to measure the photon distribution on the detection plane will, in part, determine the quality of the resulting image. A large area photon detection plane simultaneously measures both the position of the photon interaction (in three dimensions to avoid parallax effects) and the energy lost by the photon as a result of that interaction. The most notable satellite applications of this technique are GRANAT/SIGMA ${ }^{23}$ BeppoSAX, ${ }^{24}$ INTEGRAL ${ }^{14,25}$ and the soon-to-be-launched SWIFT, ${ }^{13,26}$ all of which follow in the pioneering footsteps of balloon-borne instruments such as DGT $^{27}$ and GRIP. ${ }^{28,29}$

Several interrelated parameters must be considered in designing a coded aperture imaging system. The angular resolution corresponds to the angular size of a mask element as seen from the detection plane, and so is dictated by the mask element size and the separation of the mask from the detector. The mask-detector separation is constrained by the size of the launch vehicle fairing and the FoV requirements. Assuming a mask-detector separation of $1.5 \mathrm{~m}$, the required angular resolution of 3-5 arcmin corresponds to a mask element size of 1.3-2.2 mm. The detection plane must be able to resolve the individual mask elements in the projected pattern. The exact ratio between detector spatial resolution and mask element size has an important effect on the $\mathrm{S} / \mathrm{N}$ in the reproduced image. ${ }^{30,31}$ For a fixed mask element size, improved spatial resolution results in an improvement in the imaging S/N. Jupp et al. ${ }^{31}$ showed that, to optimize the $\mathrm{S} / \mathrm{N}$ using discrete detector elements, the mask element size (in both $\mathrm{x}$ and $\mathrm{y}$ ) should be $\sim 1.5$ times larger 
than the detector spatial resolution. This implies a detector spatial resolution $(1 \sigma)$ of $0.9-1.5 \mathrm{~mm}$ in both $\mathrm{x}$ and $\mathrm{y}$ after correcting for parallax (cross-talk) effects.

The size of the fully-coded FoV, which will dictate the number of imagers required for CASTER, is determined by the geometric area of the detector and the mask-detector separation. Several factors will, however, limit the FoV. The most important of these are the mask and detector element geometries. The mask thickness must be sufficient to attenuate photons (hence, modulate the incident flux) in the desired energy range. On the other hand, the thickness of the mask must be limited to maintain uniformity of mask transmission for off-axis sources and avoid the resulting vignetting effects. Another important consideration for a large FoV instrument is the lateral distance traveled by a photon in the detector material before it interacts. This will depend not only on the angle of incidence of the photon, but also on its energy (i.e., its mean free path). The concern is that the photon may register in a detector element other than the element that it first encountered. This "pixel crosstalk" effect can seriously degrade the imaging performance. It can be minimized at a given energy by limiting the FoV (the photon incidence angle) or the detector thickness. ${ }^{32}$ An effective way to ameliorate these limitations is to measure the depth of the interaction site within the detection plane. This permits the differentiation of "deep" interactions (where the measured position may be displaced from the entrance point) from "shallow" interactions near the top of the detector (where the crosstalk is small and the measured interaction point is a good measure of the actual entrance location). This information can then be used in constructing the image. The ability to determine the depth of photon interaction is a key requirement.

The combined requirement of fine angular resolution, wide FoV, and broad energy range places severe requirements on the parameters of the coded mask. In the case of CASTER, the requirements are more challenging that any previous coded mask that has been used in space (e.g., SIGMA, Swift). In particular, we require a large-area mask with an element size of $\sim 2 \mathrm{~mm}$ and a thickness of $\sim 5 \mathrm{~mm}$ (one mean free path in tungsten at $600 \mathrm{keV}$ ). In order to attenuate the tungsten fluorescence photons, a graded mask is necessary. The closest example to what will be required is the mask that has been fabricated for the Swift mission, ${ }^{33}$ which represents the largest coded mask to date $\left(2.6 \mathrm{~m}^{2}\right)$. The Swift mask consists of $\sim 50,000$ individual lead tiles, each $5 \times 5 \mathrm{~mm}^{2}$ in area by $1 \mathrm{~mm}$ thick. The experience with Swift may provide a basis for the fabrication of the CASTER mask with its much smaller and thicker mask elements, although we must not rely on that approach. For example, we have previously developed and tested the procedures for producing

large coded aperture tungsten masks using straightforward photolithographic etching techniques. ${ }^{32,34} \mathrm{We}$ must also investigate the various options for the grading material(s) and thicknesses. ${ }^{35}$ We will identify candidate approaches to mask design and fabrication and will incorporate them into instrument simulation and mission cost trade studies.

\subsection{Detector requirements}

Given the mission requirements of a BHFP, we can compile a set of detector requirements for the coded aperture imagers that make up the CASTER instrument suite as follows:

STOPPING POWER: To provide significant stopping power at energies up to $600 \mathrm{keV}$, the detector must be composed of a high-Z, high-density material, so that the detector can be made as thin as possible.

DETECTOR SPATIAL RESOLUTION: Angular resolution requirements imply a spatial resolution $(1 \sigma)$ on the order of 0.9-1.5 mm in all three dimensions. For multi-scatter events, it is necessary to identify these events and, at the same time, measure the location and energy deposit at each site. This will help to identify the initial interaction site (for imaging) and will also allow polarization studies.

AVAILABILITY AND COST: To achieve the specified sensitivity, significant areas of detector material will be needed (up to $\sim 8 \mathrm{~m}^{2}$ ). The ability to fabricate quality detectors in such large areas within budget is therefore an important consideration.

ENERGY RESOLUTION: Although energy resolution is a desirable scientific goal, it is not critical to the success of a black hole survey. The moderate energy resolution of $\mathrm{NaI}(\sim 7 \% \mathrm{FWHM}$ at $662 \mathrm{keV})$ is sufficient to achieve the primary science goals of the BHFP. Better energy resolution, such as that afforded by $\mathrm{LaBr}_{3}(\sim 3 \% \mathrm{FWHM}$ at $662 \mathrm{keV})$ would significantly enhance the secondary science goals. 
ENVIRONMENTAL TOLERANCE: One important demand of any orbiting detector in this energy range is immunity to radiation, such as that in the earth's radiation belts. The extent to which the detector assembly can be designed to meet applicable environmental constraints (vibration, thermal, etc.) is another important constraint to be considered.

Traditional scintillation detectors offer the advantage of proven technology at relatively low cost. We have therefore studied detector configurations based on inorganic scintillators and identified $\mathrm{LaBr}_{3}$ scintillator as an excellent candidate for the CASTER detection plane. Although other inorganic scintillators (such as NaI and CsI) may possibly be used to achieve the primary science objectives, we will investigate the performance and applicability of $\operatorname{LaBr}_{3}$ given its superior energy resolution and timing characteristics.

\subsection{Lanthanum Bromide Scintillator}

Lanthanum bromide $\left(\mathrm{LaBr}_{3}\right)$ is an attractive and promising candidate for applications utilizing traditional scintillator methods. When doped with cerium it has the properties of high light output, high stopping efficiency, fast response, good linearity, and low cost. The key features of $\mathrm{LaBr}_{3}$ are listed in Table 1, along with those of other materials commonly used for hard $\mathrm{X}$ - and $\gamma$-ray detection.

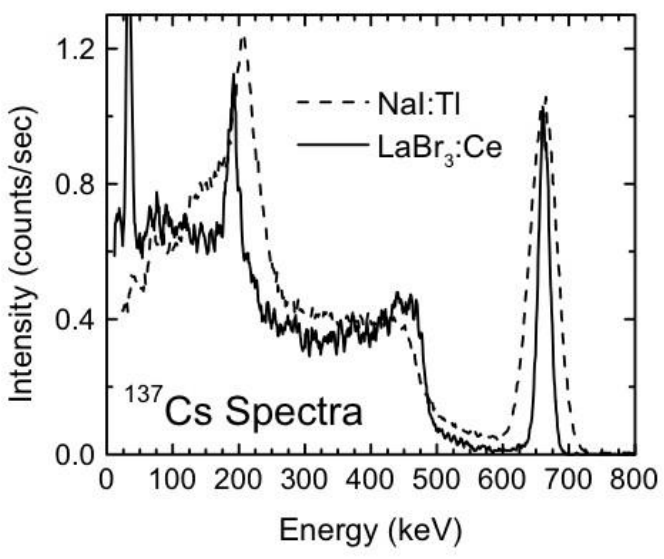

Figure 1. Energy spectrum of a ${ }^{137} \mathrm{Cs}$ source obtained with a $1 \mathrm{~cm}^{3} \mathrm{LaBr}_{3}$ detector. FWHM at 662 $\mathrm{keV}$ is $2.6 \%$. The comparison $\mathrm{Nal}(\mathrm{TI})$ spectrum has an energy resolution of $6.7 \%$ (FWHM).

The light output of $\mathrm{LaBr}_{3}$ doped with $0.5 \% \mathrm{Ce}$ is $\sim 60,000$ photons $/ \mathrm{MeV} .^{36,37}$ This light output is among the highest values for inorganic scintillators. ${ }^{38}$ In addition to the scintillation light output, the proportionality of light yield as a function of energy also contributes to the energy resolution. ${ }^{39}$ This is especially important at energies where one or more Compton interactions occur before the energy is fully absorbed. Over the energy range from 60 to $1275 \mathrm{keV}$, the non-proportionality in light yield is about $6 \%$ for $\mathrm{LaBr}_{3}$ as compared to $\sim 20 \%$ for $\mathrm{NaI}(\mathrm{Tl})$ and $\mathrm{CsI}(\mathrm{Tl}) .{ }^{36}$ With its higher proportionality and higher light output, $\mathrm{LaBr}_{3}$ provides better energy resolution than any other scintillator. Figure 1 shows the spectrum of a ${ }^{137} \mathrm{Cs}$ source obtained with a $1 \mathrm{~cm}^{3} \mathrm{LaBr}_{3}$ detector at room temperature. ${ }^{40}$ The energy resolution at 662 $\mathrm{keV}$ is $2.6 \% \mathrm{FWHM}$. This result is comparable to the quoted energy resolution (3\% @ $662 \mathrm{keV})$ for off-the-shelf spectroscopy grade CZT material from eV Products (http://www.evproducts.com/) and is also comparable to the spectral resolution of the Swift CZT array. The high signal level relative to noise with $\mathrm{LaBr}_{3}$ will permit lower energy thresholds than possible with other scintillators. Although not optimized for its low energy response, the spectrum in Figure 1 clearly shows the $\sim 32 \mathrm{keV}$ line from Ba K X-rays.

\begin{tabular}{|c|c|c|c|c|c|c|c|c|}
\hline \multicolumn{1}{|c|}{ Table 1. Summary of Detector Characteristics } \\
\hline & LaBr $_{3}$ & LaCl $_{3}$ & Nal(TI) & CsI(TI) & CsI(Na) & BGO & CZT & Ge \\
\hline Density $\left(\mathrm{g} / \mathrm{cm}^{3}\right)$ & 5.29 & 3.86 & 3.67 & 4.51 & 4.51 & 7.13 & 5.78 & 5.33 \\
\hline Light Output $(\mathrm{ph} / \mathrm{MeV})$ & 63,000 & 49,000 & 39,000 & 52,000 & 39,000 & 9000 & N/A & N/A \\
\hline$\Delta E / E(F W H M) @ 662 \mathrm{keV}$ & $<3 \%$ & $3.5 \%$ & $7 \%$ & $10 \%$ & $7.5 \%$ & $>10 \%$ & $<3 \%$ & $0.3 \%$ \\
\hline Peak $\lambda(\mathrm{nm})$ & $358-385$ & $330-352$ & 415 & 550 & 420 & 480 & N/A & N/A \\
\hline Fast Decay $(\mathrm{ns})$ & 25 & 25 & 230 & 1000 & 630 & 300 & N/A & N/A \\
\hline Hygroscopic & yes & yes & yes & slightly & yes & no & no & no \\
\hline Cost $\left(\right.$ per $\left.\mathrm{cm}^{3}\right)$ & $\$ 30$ & $\$ 30$ & $\$ 2$ & $\$ 4.50$ & $\$ 4.50$ & $\$ 9$ & $\$ 3000$ & $\$ 500$ \\
\hline
\end{tabular}


The emission spectrum of $\mathrm{LaBr}_{3}$ is well matched to the peak quantum efficiency (25\%) of borosilicate glass window PMTs with bialkali photocathodes. The spectral match to such tubes is even better than that of NaI(Tl), with roughly $20 \%$ better photocathode quantum efficiency using $\mathrm{LaBr}_{3}$. Fashioned into a position-sensitive focal plane detector for a hard X-ray camera, the high light yield of $\mathrm{LaBr}_{3}$ will translate directly into improved spatial resolution.

The fluorescent decay times of $\mathrm{LaBr}_{3}$ samples were measured using the delayed coincidence method. ${ }^{36}$ All samples, independent of Ce doping concentration, showed fast principal decay constant $(\leq 25 \mathrm{~ns})$. This assures performance superior to that possible with more slowly decaying scintillators such as NaI(Tl), CsI or BGO (Table 1) in high countrate situations, where the smaller dead-time per event becomes a distinct advantage. This may also be an advantage in efforts to reduce background via an active anticoincidence shield. In the same study, coincidence timing resolution of $\mathrm{LaBr}_{3}$ crystals with various $\mathrm{Ce}$ concentrations was measured with $511 \mathrm{keV}$ positron annihilation $\gamma$-ray pairs (emitted by

a ${ }^{22} \mathrm{Na}$ source). Measurements with a $\mathrm{LaBr}_{3}$ crystal having a $5 \% \mathrm{Ce}$ concentration yielded a FWHM timing resolution of $260 \mathrm{ps}^{36}$ This result is about a factor of three better than the timing resolution of $\mathrm{NaI}(\mathrm{Tl})$ detectors used in coincidence with plastic scintillators. In general, these results confirm that $\mathrm{LaBr}_{3}$ is well suited for applications requiring fast response and good timing resolution.

The ability to fabricate $\mathrm{LaBr}_{3}$ in large volumes would offer the opportunity to provide improved detection efficiency at higher energies. Figure 2 shows data derived from simulations of detectors having a circular area with $1 \mathrm{~cm}$ radius. The plots compare the data for $5 \mathrm{~mm}$ thick CZT with both $5 \mathrm{~mm}$ and $20 \mathrm{~mm}$ thick $\mathrm{LaBr}_{3}$. A thicker $\mathrm{LaBr}_{3}$ detector can provide better sensitivity than a $5 \mathrm{~mm}$ thick CZT-based system, especially at energies above $\sim 100 \mathrm{keV}$ (assuming comparable background levels). Improved detection plane sensitivity may also reduce the requirement on mask thickness. We will be exploring the trade-offs between detector thickness, mask thickness, background, total mass and sensitivity as part of our mission concept development.
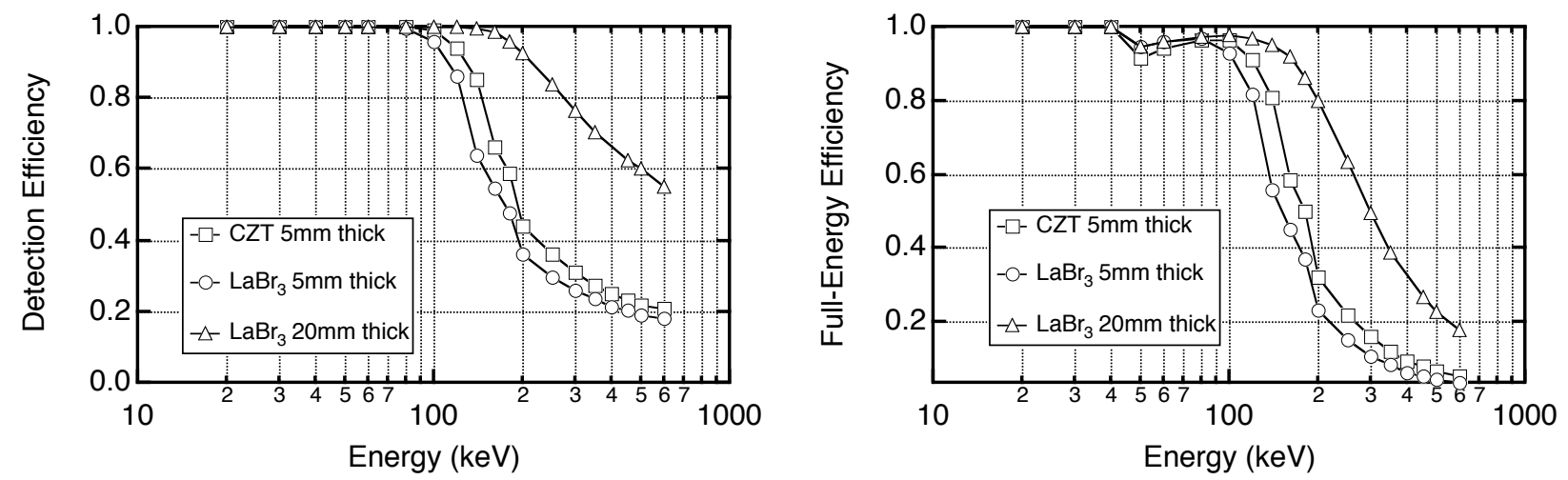

Figure 2. The total detection efficiency and the full-energy (photopeak) efficiency for circular detector volumes of $1 \mathrm{~cm}$ radius and various thicknesses.

Several experienced detector manufacturers, including RMD and Saint-Gobain Crystals and Detectors (previously Bicron), are currently growing both $\mathrm{LaCl}_{3}$ and $\mathrm{LaBr}_{3}$ crystals using proprietary processes. While 3-inch diameter $\mathrm{LaCl}_{3}$ crystals have been produced by Saint-Gobain, the crystal sizes of the denser $\mathrm{LaBr}_{3}$ material are currently limited to a few $\mathrm{cm}^{3}$. The manufacturers' early measurements are promising and, driven by the medical imaging market, they are investing heavily in the manufacturing processes for larger volumes. $\mathrm{LaCl}_{3}$ development efforts lead those of $\mathrm{LaBr}_{3}$ because it is slightly easier to work with. No fundamental barriers have been identified that would prevent crystal growth and detector fabrication with material volumes as large as are presently possible for $\mathrm{NaI}(\mathrm{Tl})$.

We know of no studies to date of $\mathrm{LaBr}_{3}$ imaging performance or radiation tolerance. These are two issues that we will be actively studying. Measurements of radiation tolerance will be especially important, given the radiation background that is experienced by space-based detectors. 


\subsection{Imaging Detector Arrays}

Gamma cameras, a.k.a. Anger cameras, imaging detector arrays formed with multiple light sensors viewing a layer of scintillation material, are widely used for medical imaging and other applications. ${ }^{41-42}$ Most commercially available gamma cameras employ layers of $\mathrm{NaI}(\mathrm{Tl})$ scintillator viewed by arrays of photomultiplier tubes (PMTs). The interaction location is determined for each detected photon from the relative signals recorded for each PMT in the array. The spatial resolution of a gamma camera depends on geometrical factors such as the scintillator thickness, its size and the number and density of the light sensors in the array. Statistical and optical factors, such as scintillation yield, surface reflectivity, spectral match and photoelectron yield are also important. ${ }^{15}$ New gamma cameras employing the combination of higher light yield scintillator material $\left(\mathrm{LaBr}_{3}\right)$ and a higher density of light sensors, (e.g., small element MAPMTs) will have significantly better spatial resolution capabilities over earlier versions. Four examples of demonstrated performance with gamma cameras across the applicable range of detector thickness follow. Note that, in each of these examples, the PMT diameter and/or pitch is approximately equal to or greater than the thickness of the scintillator layer.

Example 1: Spatial resolution $(1-\sigma)$ of $1-2 \mathrm{~mm}$ at $60 \mathrm{keV}$ was demonstrated at NRL using a $4 \mathrm{~mm}$ thick monolithic layer of $\mathrm{CsI}(\mathrm{Tl})$ coupled to a crossed-wire position-sensitive photomultiplier tube (PSPMT). ${ }^{43}$ The PSPMT, Hamamatsu R2487, employed in the NRL study features a bialkali photocathode and crossed wire anodes spaced on 3.75 and 3.70 $\mathrm{mm}$ pitch in $x$ and $y$ respectively. This fine spatial resolution performance achieved in the NRL study is noteworthy given the spectral mismatch between $\mathrm{Csl}(\mathrm{Tl})$ and bialkali photocathodes. This combination typically results in a $\sim 49 \%$ reduction in photoelectron yield for $\gamma$-rays with respect to that of $\mathrm{NaI}(\mathrm{Tl})$ (Table 1). ${ }^{38} \mathrm{We}$ can extrapolate these measurements to the position-resolution performance expected for the same thickness of a monolithic layer of $\mathrm{LaBr}_{3}$ viewed by this PSPMT. The photoelectron yield with $\mathrm{LaBr}_{3}$ scintillator will be $3.3 \times$ that in the NRL configuration (Table 1). Noting that position resolution for Anger cameras varies as the inverse square root of the yield, ${ }^{42}$ we expect an improvement of at least $1.8 \times$ in the position resolution or $0.6-1.1 \mathrm{~mm}(1-\sigma)$ at $60 \mathrm{keV}$. Furthermore, unlike an anode wire in a PSPMT, where $x$ - and $y$-dimension wires must share the signal, each MAPMT pixel provides a simultaneous measure of both the $x$-and $y$-coordinate, further increasing the effective yield from that of the NRL configuration.

Example 2: An $11 \mathrm{~mm}$ thickness of $\mathrm{LaBr}_{3}$ will have the same stopping power for $511 \mathrm{keV}$ photons as a $10 \mathrm{~mm}$ thickness of CZT. This thickness is close to that of commercial medical Anger cameras that have $9.5 \mathrm{~mm}$ thick layers of $\mathrm{NaI}(\mathrm{Tl})$ and arrays of $25 \mathrm{~mm}$ or $76 \mathrm{~mm}$ diameter (single anode) PMTs. Typical spatial resolution (1- $\sigma$ ) is $1.5 \mathrm{~mm}$ at $141 \mathrm{keV}$. A $27 \%$ improvement in spatial resolution or $1.2 \mathrm{~mm}(1-\sigma)$ is expected with $\mathrm{LaBr}_{3}$ based solely on increased photoelectron yield. This level of spatial resolution matches that required for CASTER. A further improvement in spatial resolution from a higher density of scintillation light sensors may also be possible. To help maintain spatial resolution across a broad energy range energy range, thin slots, cut partially into the scintillator at the PMT surface and filled with reflective material, are sometimes used to control light distribution in thick crystals. Such slotted crystals (e.g. Bicron StarBrite) have become common in medical imaging applications, because they can serve the dual use functions of SPECT $(141 \mathrm{keV})$ and PET $(511 \mathrm{keV})$ imaging.

Example 3: The detection plane of the orbiting SIGMA telescope ${ }^{23}$ was an Anger camera using a $12.5 \mathrm{~mm}$ thick layer of $\mathrm{NaI}(\mathrm{Tl})$. The total area of the scintillator $\left(2550 \mathrm{~cm}^{2}\right)$ was covered with an array of close-packed hexagonal PMTs. The spatial resolution varied between $2.5 \mathrm{~mm}$ at a few hundred $\mathrm{keV}$ to $\sim 5 \mathrm{~mm}$ at $30 \mathrm{keV}$. We expect a $27 \%$ improvement in spatial resolution based on the photoelectron yield with $\mathrm{LaBr}_{3}$.

Example 4: The mean free path of the most penetrating photons $(\sim 5 \mathrm{MeV})$ is $7.8 \mathrm{~cm}$ in NaI(Tl) and $5.6 \mathrm{~cm}$ in $\mathrm{LaBr}_{3}$. The Gamma-Ray Imaging Paylod (GRIP) employed $41 \mathrm{~cm}$ diameter, $5 \mathrm{~cm}$ thick NaI(Tl) detectors read out by $197.6 \mathrm{~cm}$ diameter PMTs, each separated by $8.3 \mathrm{~cm}$ from its neighbors. The researchers measured a $1-\sigma$ spatial resolution of 0.5 $\mathrm{cm}$ at $122 \mathrm{keV}$ and $0.3 \mathrm{~cm}$ at $662 \mathrm{keV}{ }^{28}$ A detector formed of $\mathrm{LaBr}_{3}$ in this same geometry would have greater stopping power as well as higher yield. We expect a $27 \%$ improvement in spatial resolution based on the photoelectron yield with $\mathrm{LaBr}_{3}$.

Table 3 summarizes these four examples. The measured 1-d spatial resolution $(1-\sigma)$ ranges from $6 \%$ to $40 \%$ of the scintillator thickness. The best ratios are obtained with large area $\mathrm{NaI}(\mathrm{Tl})$ detectors and bialkali PMTs. The poorest 
ratio corresponds with the lowest yield configuration, $\mathrm{CsI}(\mathrm{Tl})$ with a bialkali PSPMT. Using $\mathrm{LaBr}_{3}$ we can expect an improvement in spatial resolution based solely on increased light output. With all other parameters being equal, the position resolution for Anger cameras varies as the inverse square root of the light yield (Macovski 1983; Bouchet et al. 2001). Simply replacing $\mathrm{CsI}(\mathrm{Tl})$ or $\mathrm{NaI}(\mathrm{Tl})$ with $\mathrm{LaBr}_{3}$ in any of the above configurations will result in a $27 \%$ improvement in spatial resolution (in addition to a significant improvement in stopping power). The estimated spatial resolution resulting from a simple replacement with $\mathrm{LaBr}_{3}$ is shown in the fifth column of Table 2. These numbers are conservative in that they do not reflect the improvements that can be expected from the use of alternative light sensors and light sensor arrangements. In some cases, however, we have already reached the required spatial resolution (0.9-1.5 $\mathrm{mm})$. Note also that the expected improvement with $\mathrm{LaBr}_{3}$ is computed without considering the spectral match advantage it has over $\mathrm{NaI}(\mathrm{Tl})$ with bialkali photocathodes. With proper calibration and analysis of the multiple PMT signals, the achievable energy threshold and spectroscopic performance of these Anger cameras would be similar to that achieved using single PMT spectrometers. ${ }^{28,43}$

\begin{tabular}{|c|c|c|c|c|}
\hline \multicolumn{5}{|c|}{ Table 2. Anger Camera Examples } \\
\hline & Energy & Thickness & $\sigma_{x, y}$ (avg.) & $\sigma_{x, y}\left(w / L a B r_{3}\right)$ \\
\hline CsI(TI)/PSPMT (NRL) & $60 \mathrm{keV}$ & $4 \mathrm{~mm}$ & $1.5 \mathrm{~mm}$ & $0.8 \mathrm{~mm}$ \\
\hline $\operatorname{Nal}(T I)$ (Medical) & $141 \mathrm{keV}$ & $9.5 \mathrm{~mm}$ & $1.5 \mathrm{~mm}$ & $1.2 \mathrm{~mm}$ \\
\hline $\mathrm{NaI}(\mathrm{TI})(\mathrm{SIGMA})$ & $30 \mathrm{keV}-1 \mathrm{MeV}$ & $12.5 \mathrm{~mm}$ & $2.5-5.0 \mathrm{~mm}$ & $2.0-4.0 \mathrm{~mm}$ \\
\hline $\operatorname{Nal}(T I)(G R I P)$ & $662 \mathrm{keV}$ & $5 \mathrm{~cm}$ & $3.0 \mathrm{~mm}$ & $2.3 \mathrm{~mm}$ \\
\hline
\end{tabular}

In addition to providing the location in the $x$ - and $y$-dimensions, the extent of the distribution of scintillation signals within the sensor plane provides a measure of the $z$-coordinate or depth of the interaction. Light from scintillations nearer the sensor plane is shared among fewer sensors than light from scintillations farther from the sensor plane. Figure 3 illustrates the $z$-coordinate measurement principle. The GRIP experiment demonstrated that selection on the depth parameter allowed $87 \%$ rejection of $122 \mathrm{keV}$ photons flood illuminating the back of the detector while allowing $90 \%$ acceptance of photons from the front. The depth resolution varied dramatically from a few $\mathrm{mm}$ at the center of the PMT near the sensor plane to little or no depth information near the intersection between 3 PMTs. The GRIP study concluded that that the depth resolution could be improved and made more uniform by employing a larger number of smaller PMTs. ${ }^{28}$ Anger cameras with a higher density of readout sensors (with diameter and/or pitch less than the thickness of the scintillator layer) will have improved ability

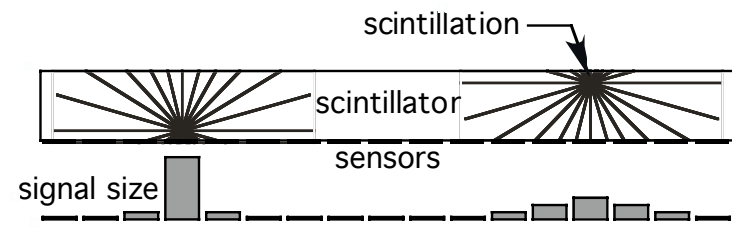

Figure 3. Measurement of the interaction depth. to measure the $\mathrm{z}$ coordinate.

Hamamatsu has developed a series of MAPMTs with individual anodes as small as $2 \mathrm{~mm}$. Of particular interest is a compact, low profile flat panel MAPMT (H8500), designed specifically for large area arrays that is currently available in an $8 \times 8$ anode configuration with $5.6 \mathrm{~mm}$ anodes $^{44}$; smaller anode versions are under development. Burle has recently produced a similar device (the Planacon ${ }^{\mathrm{TM}}$ tube), based on potentially more rugged MCP technology, with a very similar geometry. In either case, arrays of these devices have very little dead space $(\leq 10 \%)$. Several types of silicon photo-detectors could also be considered as viable readout options. ${ }^{45}$ The available signal will generally be split among several sensors. Interpolation among the triggered sensors will provide the needed spatial resolution, with the sensor size providing an upper bound on the spatial resolution at all energies. Anger cameras with a higher density of PMTs would have an improved ability to measure the $z$ coordinate. Multi-hit events would be identified and the interaction site locations measured in those cases where spatial resolution is better than the mean free path of the scattered photons. This advantage is important for imaging above $\sim 250 \mathrm{keV}$ where the fraction of Compton-scattered photons is greater. This will also open up the possibility of polarization studies with CASTER.

The use of a higher density of smaller sensor elements requires a larger number of processing channels that can be handled by the development of a suitable low power ASIC. Image plane detectors formed with scintillators and 
MAPMTs will have significant power advantages over those formed with solid-state detectors. The biasing of MAPMTs will require $<1 \mathrm{~mW} / \mathrm{cm}^{2}$ of detector area. In addition, MAPMTs also provide high gain with little noise, thus reducing the preamplifier power required for each front-end electronics channel as compared to solid-state detectors.

The power requirements can also be reduced by a reduction in the number of electronics channels. The number of electronics channels for a CZT pixel detector or Anger camera/MAPMT combination is on the order of $n_{x} n_{y}$, where $n_{x}$ and $\mathrm{n}_{\mathrm{y}}$ are the number of sensor elements in the $\mathrm{x}$ - and y-directions respectively. For an $8 \mathrm{~m}^{2}$ total detector area covered by $5 \mathrm{~mm}$ MAPMT pixels, this amounts to $3.2 \times 10^{5}$ detector elements and a corresponding number of electronics channels. The number of electronics channels can be reduced to the order of $\mathrm{n}_{\mathrm{x}}+\mathrm{n}_{\mathrm{y}}$ by the use of a "crossed fiber" readout approach adapted from medical imaging applications ${ }^{46,47}$ and analogous to the design of solid state strip detectors. In this case, one layer of wavelength-shifting fibers is laid in the x-direction across the top of a scintillator and a second layer of fibers is laid in the y-direction across the bottom. The light emitted by the fibers is read out at one end of each fiber by a set of MAPMTs. The crossed fiber layers measure the $\mathrm{x}$ - and $\mathrm{y}$ - position using the center of gravity of the light in the two fiber arrays, and the depth by using the signal distribution across the fiber arrays (Figure 3). Only a small fraction of the light is absorbed, reemitted, and trapped in the fibers, however. Most of the light escapes the fibers. The energy measurement, therefore, is performed by a set of "energy measuring" PMTs viewing the scintillator through the bottom fiber layer. Unfortunately, the spectral match of these fibers with $\mathrm{LaBr}_{3}$ is not ideal. Some compensation may be possible due to the higher light output of the $\mathrm{LaBr}_{3}$, but this remains to be demonstrated. We will be using detector simulations to study the suitability of the crossed fiber approach for $\mathrm{LaBr}_{3}$.

In laboratory tests at LSU, we have used segmented scintillator arrays produced by St. Gobain so that the light is concentrated in a small number of fibers (an approach that

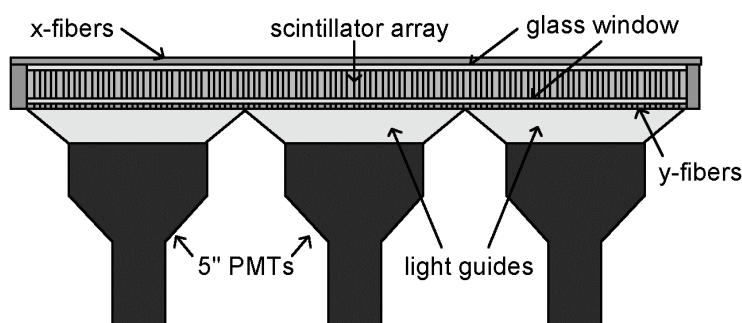

Figure 4. An imager design consisting of pixellated scintillator array read out by wavelength shifting fibers. The large area PMTs are usded for measuring total energy deposit. may be especially effective at lower energies where the scintillator output is reduced). Individual $2 \mathrm{~mm} \mathrm{CsI(Na)}$ elements are grown, cut to length, polished, enclosed in reflective wrappers, and assembled into the full array. With an absorption peak that matches the emission peak of the $\mathrm{CsI}(\mathrm{Na})$ at $420 \mathrm{~nm}$, the fibers effectively absorb the scintillation light and re-emit a portion of it down the fiber axis, where it is read out by a MAPMTs. A set of 5-inch PMTs are used to measure the total light output on one side of the scintillator array as a means of measuring total energy deposit. The measured energy resolution (FWHM) with a CsI(Na) scintillator and $1 \mathrm{~mm}$ fibers is $15 \%$ at $60 \mathrm{keV}$. A schematic of this setup is shown in Figure 4.

\section{MISSION ARCHITECTURE}

Our CASTER mission concept closely parallels that of EXIST. ${ }^{12}$ The EXIST concept is rather mature, having been through two GSFC design studies. The most significant change implied by the CASTER concept is in the design of the imaging system. In particular, CASTER envisions coded aperture detection planes based on some type of scintillatorbased detection system (such as $\mathrm{LaBr}_{3}$ ) rather than CZT. This difference will have implications for the imaging system, the detector background, the mask fabrication, power requirements and spacecraft weight. All of these issues (and perhaps others) are being reconsidered in the CASTER mission concept study. The essential mission architecture, however, would remain largely unchanged.

CASTER would rely on an array of coded aperture imagers to scan the full sky once every orbit, with a FoV that ideally would extend the full $180^{\circ}$ from pole to pole. The precise number, size and arrangement of coded aperture imagers would be determined by the concept study. Alternatively, a somewhat smaller FoV could be used in a "rocking" configuration that would effectively cover the full $180^{\circ}$ to achieve full-sky coverage. The array would be designed to 
provide the required sensitivity and angular resolution over the energy range from 10 to $600 \mathrm{keV}$. A zenith-pointed mode would be the baseline for the survey part of the mission, lasting perhaps one year. Once the survey is complete, a pointed operation mode may be appropriate for maximizing the exposure to selected sources. The pointing requirements are fairly modest with a pointing stability of $\sim 0.5^{\circ}$ and aspect knowledge to $\sim 1$ arcmin. The scanning mode of operation offers the added benefit of dealing with the non-uniformities in the detector background by effectively smoothing out the resulting image noise. (INTEGRAL uses a dithering process to achieve the same result.) Detector collimation may be required at lower energies to reduce the impact of the cosmic diffuse emission, which dominates the background at energies below $\sim 100-200 \mathrm{keV}$. A combination of active and passive shielding will be used for the detector array to minimize the effects of cosmic induced background and Compton suppression. The details of the collimation detector shielding will be developed as part of the simulation effort. Payload mass, size and power are important in choosing a suitable launch vehicle. The orbit will affect the background and thus the sensitivity. The preferred orbit would be a low-Earth equatorial orbit with a minimum two-year lifetime. Having an equatorial orbit would provide the lowest possible $\gamma$-ray background and therefore the highest sensitivity. The HETE- 2 mission has demonstrated the value of such a low-background orbit for high energy detectors.

To maximize the scientific return from the mission, the data will be returned on an event-by-event basis. Each event will be time tagged with an accuracy of $\sim 10 \mu \mathrm{s}$. Multi-hit information will also be returned with each event to help construct the image and for polarization studies. A dedicated burst response mode will be developed to provide prompt on-board processing of $\gamma$-ray burst data. The on-board generation of appropriate sky maps would facilitate the rapid dissemination of burst locations to the astronomical community, as in the case of BATSE and HETE-2 (and the upcoming Swift mission). The burst mode would also insure that the data are recorded in sufficient detail for groundbased analysis. Once the data are on the ground, rapid processing and release of the data to the public would enable ground-based follow-ups. A guest observer facility is envisaged to handle data dissemination and to provide support to qualified guest investigators.

\section{SUMMARY}

Inorganic scintillator technology is well established in space-based applications. $\mathrm{LaBr}_{3}$ is a promising new scintillator material. It has high stopping power, high light output, fast response, and shows good energy and timing resolution. All studies to date indicate that these properties are maintained as the crystal volume is increased. Undoubtedly the spatial resolution capabilities of gamma cameras made with $\mathrm{LaBr}_{3}$ will be better than present day NaI or CsI-based instruments, but this remains to be demonstrated and the improvement measured. The extent to which a higher density of scintillation light sensors improves the uniformity, 3-d spatial resolution, and multi-hit recognition capabilities of the detectors requires further study. In addition, a detailed demonstration of the crossed fiber performance, including position and energy resolution over the full range of incident photon energies and incidence directions, must be demonstrated. It will be important to further characterize detector capabilities, develop and validate $\mathrm{LaBr}_{3}$ simulation models, and assess the impact of radiation exposures before defining optimum instrument configurations. Despite the promise of $\mathrm{LaBr}_{3}$, we will continue to investigate other more traditional scintillator options in the context of CASTER.

There are several technical issues that are being addressed as part of the CASTER mission concept study. We recognize the following as the most important technology issues to focus on, all of which should be resolved within the time frame projected for the Einstein Probe missions:

1) CONTINUED DEVELOPMENT OF LABR3. Although the manufacturers are currently very optimistic that this goal can be achieved, it cannot be considered a certainty until such volumes are actually produced.

2) EVALUATION OF SCINTILLATOR-BASED IMAGERS. We will be simulating the performance characteristics of gamma cameras based on various inorganic scintillators (concentrating on $\mathrm{LaBr}_{3}$ ), studying both the Anger camera configuration and the crossed fiber readout design. Simulations will also help to develop methods for depth measurement within the scintillator as a guide for future laboratory studies. 
3) IMAGER DESIGN. The imaging design will have to be carefully developed, taking into account the many interrelated factors, such as detector thickness, sensitivity, mask element size, mask detector separation, background, etc. Various mask fabrication options must be considered and demonstrated before a flight-ready mask can be produced.

4) BACKGROUND STUDIES. Very little is currently known about what background of $\mathrm{LaBr}_{3}$ in an orbital radiation environment. We plan to simulate the background using MGGPOD as part of our concept study, but measurements of the $\mathrm{LaBr}_{3}$ background on balloon platforms and perhaps also on orbit will be needed before it can reach an advanced state of flight-readiness.

5) SENSOR RUGGEDIZATION. For many of the sensors being considered for CASTER, the ruggedness for spaceflight use has not yet been demonstrated.

6) DATA HANDLING. Suitable low-power ASICs must be available for handling the large number of signal channels from the readout sensors. On-board event triggering algorithms must be developed to maximize the scientific return within the telemetry limitations.

\section{REFERENCES}

1. Grindlay, J. et al., "EXIST: The Ultimate Spatial/Temporal Hard X-Ray Survey," GAMMA 2001, S.Ritz, N. Gehrels \& C.R. Shrader, ed., AIP Conf. Proc. 587, pp. 899-908, AIP, New York, 2001.

2. Grindlay, J. et al., "Proposed Next Generation GRB Mission: EXIST," Gamma-Ray Burst and Afterglow Astronomy 2001, G.R. Ricker \& R.K. Vanderspek, ed., AIP Conf. Proc. 662, pp. 477-480, AIP, New York, 2003.

3. McConnell, M. L., et al., "The Soft Gamma-Ray Spectral Variability of Cygnus X-1,” Ap. J. 572, pp. 984, 2002.

4. Grove, J. E., W. N. Johnson, R. A. Kroeger, et al., "Gamma-Ray Spectral States of Galactic Black Hole Candidates," Ap. J. 500, pp. 899, 1998.

5. Purcell, W. et al., "OSSE Mapping of Galactic $511 \mathrm{keV}$ positron Annihilation Emission," Ap. J. 491, pp. 725-748, 1997.

6. Jean, P. et al., "Early SPI/INTEGRAL Measurements of $511 \mathrm{keV}$ Line Emission from the $4^{\text {th }}$ Quadrant of the Galaxy," Astron. Astrophys. 407, pp. 55-58, 2003.

7. Cassé, M., Cordier, B., Paul, J., and Schanne, S., "Hypernovae/Gamma-Ray Bursts in the Galactic Center as Possible Sources of Galactic Positrons," Ap. J. 602, pp. L17-L20, 2004.

8. Bertone, G. et al., "Gamma Ray Bursts and the Origin of Galactic Positrons," astro-ph/0405005, 2004.

9. Schanne, S, Cassé, M., Bertrand, C. and Paul, J., "Hypernovae as Possible Sources of Galactic Positrons," The INTEGRAL Universe, Proc. Of the $5^{\text {th }}$ INTEGRAL Workshop, in press, astro-ph/0404492.

10. Cassé, M., Fayet, P., Schanne, S., Cordier, B., and Paul, J., "INTEGRAL and Light Dark Matter," The INTEGRAL Universe, Proc. Of the $5^{\text {th }}$ INTEGRAL Workshop, in press, astro-ph/0404490.

11. Boehm, C., Hooper, D., Silk, J., Casse, M., and Paul, J., “MeV Dark Matter: Has It Been Detected?" Phys. Rev. Letters 92, pp. 101301-1-4, 2004.

12. Grindlay, J. E., et al., "EXIST: mission design and concept and technology program," Proc. SPIE 4851, pp. 331344, 2002.

13. Barthelmy, S. D., "The Burst Alert Telescope (BAT) on the Swift MIDEX Mission," Proc. SPIE 5165, pp. 175$188,2004$.

14. Ubertini, P., et al., "The Imager on Board INTEGRAL," Proc. SPIE 2806, pp. 246-257, 1996.

15. Harrison, F. A., S, M. Kahn, C. J. Hailey, and K. P. Ziock, "Performance Optimization for Hard X-ray / Soft $\gamma$-ray Detectors," SPIE Proc. 1344, pp. 47-52, 1990.

16. Baumgartner, W.H., et al., "The InFOC $\mu$ s Hard X-Ray Telescope: Pixellated CZT Detector / Shield Performance and Flight Results,” Proc. SPIE 4851, pp. 945-956, 2003.

17. Silver, E., $\mathrm{t}$ al., "B-MINE, The Balloon-Borne Microcalorimeter Nuclear Line Explorer," SPIE. Proc. 4851, pp. 905-912, 2003.

18. Halloin, H., et al., "Design and Flight Performance of a Crystal Diffraction Telescope," SPIE Proc. 4851, pp. 895904, 2004.

19. Harrison, F. A., et al., "The High Resolution Spectroscopic Imaging (HSI) Mission,” SPIE Proc. 4851, pp. 345$352,2003$. 
20. Boggs, S. E., et al., "The Cyclone Hard X-Ray Observatory,” SPIE Proc. 4140, pp. 166-177, 2000.

21. Caroli, E., et al., "Coded Aperture imaging in X-and Gamma-Ray Astronomy,” Sp. Sci. Rev. 45, pp. 349-403, 1987.

22. Skinner, G. K., T. J. Ponman, A. P. Hammersley, and C. J. Eyles, "Techniques for the Analaysis of Data from Coded Mask X-ray Telescopes,” Astrophys. Space Sci. 136, pp. 337-349, 1987.

23. Bouchet, L., et al., "The SIGMA/GRANAT Telecsope: Calibration and Data Reduction" Ap. J. 548, pp. 990-1009, 2001.

24. Frontera, F., et al., "The High Energy Instrument PDS On-Board the BeppoSAX X-ray Astronomy Satellite," Astron. Astrophys. Suppl. 122, pp. 357-369, 1997.

25. Parmar, A., C. Winkler, P. Barr, L. Hansson, E. Kuulkers, R. Much, and A. Orr, "The INTEGRAL Mission," SPIE Proc. 4851, pp. 1104-1112, 2003.

26. Gehrels, N., "The Swift Gamma Ray Burst MIDEX,” Proc. SPIE 4140, pp. 42-49, 2000.

27. Dunphy, P. P., et al., "A Balloon-borne Coded Aperture Telescope for Low-Energy Gamma-Ray Astronomy,” NIM A274, pp. 362-379, 1989.

28. Finger, M. H., "The Imaging of Extra-Galactic Low-Energy Gamma-Ray Source Prospects, Techniques, and Instrumentation," Ph.D. Thesis, California Institute of Technology, 1987.

29. Schindler, S. M., et al., "GRIP-2: A Sensitive Balloon-borne Imaging Gamma-Ray Telescope," NIM A384, pp. 425-434, 1997.

30. Charalambous, P. M., A. J. Dean, J. B. Stephen, and N. G. S. Young, "Aberrations in Gamma Ray Imaging Systems," NIM 221, pp. 56-59, 1984.

31. Jupp, I. D., K. Byard, and A. J. Dean, "An Improved Sampling Configuration for a Coded Aperture Telescope," NIM A345, pp. 576-584, 1994.

32. McConnell, M., et al., "A Balloon-Borne Coded Aperture Telescope for Arc-Minute Angular Resolution at Hard XRay Energies," SPIE Proc. 2806, pp. 349-360, 1996.

33. Vigneau, D. and D. W. Robinson, "Large Coded Aperture mask for Spaceflight Hard X-Ray Images," SPIE Proc. 4851, pp. 1326-1335, 2003.

34. Altice, P. P., et al., "MARGIE - Minute of Arc resolution hard X-Ray / Gamma Ray Imaging Telescope for an ultra-Long Duration Balloon Mission,” Adv. Sp. Res. 30, pp. 1321-1332, 2002.

35. Robinson, D. W., "Building a New Kind of Graded-Z Shield for Swift's Burst Alert Telescope," SPIE Proc. 4851, pp. 1374-1381, 2003.

36. Shah, K. S., J et al., "LaBr $:$ Ce Scintillators for Gamma Ray Spectroscopy," IEEE Trans. Nucl. Sci. 50, pp. 24102413, 2003.

37. van Loef, E. V. D., et al., "High Energy Resolution Scintillator: $\mathrm{Ce}^{3+}$ Activated $\mathrm{LaBr}_{3}$," Appl. Phys. Lett. 79, pp. $1573-1575,2001$.

38. Knoll, G., Radiation Detection and Measurement, John Wiley and Sons, Inc, New York, 1999.

39. Dorenbos, P., J. T. M. de Hass and C. W. E. van Ejik, "Non-Proportionality in the Scintillation Response and the Energy Resolution Obtainable with Scintillation Crystals," IEEE Trans. Nucl. Sci. 42, pp. 2190-2202, 1995.

40. Shah, K.S., et al., "High Energy Resolution Scintillation Spectrometers," IEEE NSSConf. Record, 2003.

41. Anger, H. O., "Scintillation Camera," Rev. Sci. Instrum. 29, 1958.

42. Macovski, A., Medical Imaging Systems, Prentice Hall, New York, 1983.

43. Kroeger, R. A., et al., "Thin Scintillators and Position Sensitive Photomultiplier Tubes for Hard X-ray Imaging in Space," IEEE Trans. Nucl. Sci. 44, pp. 881-884, 1997.

44. Pani, R., et al., "Evaluation of Flat panel PMT for Gamma Ray Imaging," NIM A504, pp. 262-268, 2003.

45. Fiorini, C., "Gamma detectors for Spectroscopy and Imaging Based on Scintillators Coupled to Silicon Photodetectors," SPIE Proc. 4141, pp. 97-110, 2000.

46. Matthews, K. L., S. M. Leonard, C. E. Ordonez, and W. Chang, "A Depth-Encoding Anger Detector Using Scintillating Fibers," IEEE Trans. Nucl. Sci. 48, pp. 1397-1402, 2001.

47. Matthews, K. L., S. M. Leonard, C. E. Ordonez, and W. Chang, "Comparison of Wavelength-shifting Fiber Types and Methods of Ribbon Assembly for the Depth-encoding Anger Detector," IEEE NSS Conf. Record, 2002. 